\title{
Terahertz Photovoltaic Response of Si-MOSFETs: Spin Related Effect
}

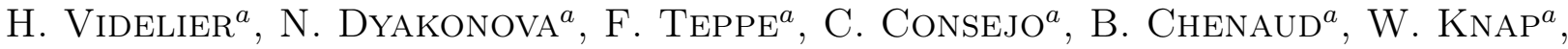 \\ J. Lusakowski ${ }^{b}$, D. Tomaszewski ${ }^{c}$, J. MARCZEWSki $^{c}$ And P. GRABIEC ${ }^{c}$ \\ ${ }^{a}$ GES-UMR5650, Université Montpellier 2 and CNRS, 34095 Montpellier, France \\ ${ }^{b}$ Institute of Experimental Physics, Warsaw University, Warsaw, Poland \\ ${ }^{c}$ Institute of Electron Technology, al. Lotników 32/46, 02-668 Warsaw, Poland
}

\begin{abstract}
We report on investigations of photovoltaic response of Si-MOSFETs subjected to terahertz radiation in high magnetic fields. Then a DC drain-to-source voltage is developed that shows singularities in magnetic fields corresponding to paramagnetic resonance conditions. These singularities are investigated as a function of incident frequency, temperature and two-dimensional carrier density. We tentatively attribute these resonances to spin transitions of the electrons bound to Si dopants and discuss the possible physical mechanism of the photovoltaic signal generation.
\end{abstract}

PACS: $85.30 . \mathrm{Tv}$

\section{Introduction}

Silicon MOSFETs have been recently shown as sensitive sub-terahertz radiation detectors at room temperature [1-3]. The photovoltaic response dependences on the gate length and the gate bias are in good agreement with the Dyakonov-Shur plasma wave detection theory [4]. The detection signal results from the rectification of high frequency currents induced by the incident radiation in the transistor channel. The rectification takes place due to a nonlinear response of the gated two-dimensional electron gas and a source-drain asymmetry. The main source of nonlinearity is the superposition of two radiation-induced effects: (i) the modulation of the carrier density in the channel by variations of the gate potential and (ii) drift velocity modulation due to variations of the drain potential. Recently, monolithically integrated $\mathrm{THz}$ focal-plane arrays including antennas and amplifiers on a single silicon die have been designed to work at room temperature [5].

In this work we have studied the effect of high magnetic field on the Si-MOSFETs photovoltaic response subjected to sub-terahertz radiation.

\section{Results}

Silicon-on-insulator (SOI) MOSFETs were used in our study. Gate length and width in these structures were $L_{\mathrm{g}}=10 \mu \mathrm{m}$ and $W_{\mathrm{g}}=5 \mu \mathrm{m}$, respectively. The transistors were bonded to a commercially available multipin support with a gold wire. The packaged devices were placed at the centre of a superconducting coil in the Faraday configuration and cooled by a helium exchange gas. Sub-terahertz radiation in the range $185-300 \mathrm{GHz}$ was brought from a backward wave oscillator source or a gunn diode to the transistor through a waveguide. The radiation was coupled to the device through metallization pads. The photovoltaic signal, $\Delta U$, was measured between source and drain contacts using a standard lock-in technique. The temperature range was from $10 \mathrm{~K}$ to $300 \mathrm{~K}$.

The photoresponse depends on the gate bias in a typical way showing a maximum close to the threshold voltage [1]. With temperature decrease the photoresponse maxima are shifted to higher gate bias following the shift of the transistor threshold. Also the amplitude of the photoresponse grows by approximately one order of magnitude with decreasing temperature from $300 \mathrm{~K}$ down to $10 \mathrm{~K}$.

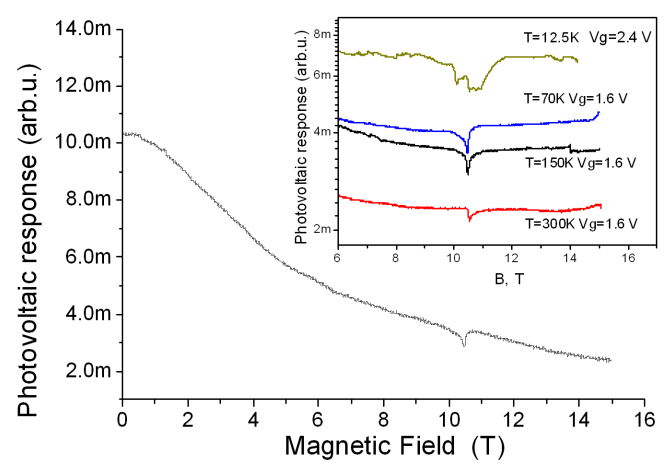

Fig. 1. Photovoltaic signal at $10 \mathrm{~K}$ as a function of applied magnetic field at incident frequency $300 \mathrm{GHz}$. Gate voltage is $1.6 \mathrm{~V}$. Inset: temperature dependence of photovoltaic response in the vicinity of singularity. 
The magnetic field affected the photoresponse in two manners (Fig. 1). First, we observed a smooth decrease of the signal with magnetic field. Secondly, at a certain magnetic field the photoresponse shows a specific feature. When the radiation frequency increases the specific feature shifts to higher magnetic fields.

The smooth decreasing behavior can be explained by plasma waves damping that takes place at magnetic fields $B$ higher than the cyclotron resonance field $B_{\mathrm{c}}=$ $\omega_{\mathrm{c}} m / e$, where $\omega_{\mathrm{c}}$ is the cyclotron frequency, $m$ is the electron effective mass. For silicon at $300 \mathrm{GHz}, B_{\mathrm{c}} \approx 2 \mathrm{~T}$ is obtained. In agreement to this estimation one can see in Fig. 1 that the signal begins to decrease significantly around $2 \mathrm{~T}$. More detailed description of the influence of magnetic field on the $\mathrm{THz}$ detection by field effect transistor can be found in Ref. [6].

The inset of Fig. 1 shows also the singularity and its evolution with temperature. One can see that at low temperature $(12.5 \mathrm{~K})$ the singularity has a complex structure, composed of several lines. With temperature increase side lines disappear, the structure as a whole narrows and its relative amplitude decreases (note the logarithmic scale of the photoresponse axis).

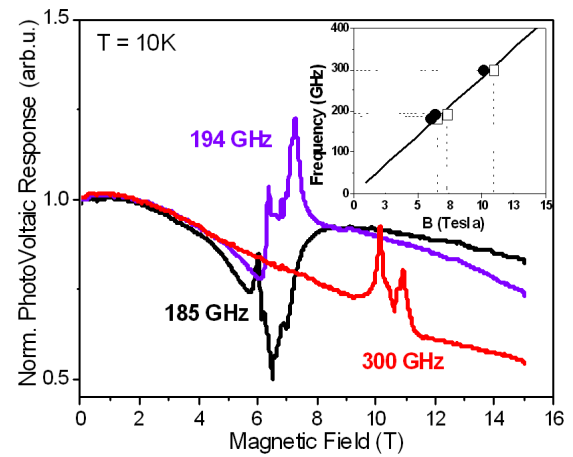

Fig. 2. Photovoltaic signal at $10 \mathrm{~K}$ as a function of applied magnetic field at three different incident frequencies: $185 \mathrm{GHz}, 194 \mathrm{GHz}, 300 \mathrm{GHz}$. Gate voltage is $2.4 \mathrm{~V}$. Inset shows the position of the 2 peaks (full circles and empty squares). Straight line is a calculation with $g=2$.

In Fig. 2, photovoltaic signal is presented at $10 \mathrm{~K}$ as a function of applied magnetic field for three different incident frequencies. A constant bias of $2.4 \mathrm{~V}$ was applied on the gate, corresponding to its threshold voltage value and hence to the transistor's maximum of sensitivity. For the sake of clarity the curves are normalized to the signal value at zero field. A very well pronounced feature is visible on each curve with two main peaks shifting to higher fields by increasing incident light frequency. The position of these peaks is plotted in the inset of Fig. 2 where the straight line is a calculation of the frequency dependence on the magnetic field with the following equation: $f=g \mu_{\mathrm{B}} B / h$ and $g=2$ corresponding to the $g$ factor of typical silicon dopants. One can see that the experimental points follow the calculated dependence indicating a possible link between the observed characteristics of the photovoltaic response and paramagnetic resonances (spin resonance transitions).

The exact physical mechanism leading to photovoltaic response related to spin resonance transitions is not clear up to now. In addition, from experimental point of view there are several unclear issues. For example, the signal can change its shape dramatically with temperature, light frequency or between sample cooling cycles (compare Figs. 1 and 2). Further experimental and theoretical investigations are needed.

Looking for theoretical explanation of the observed phenomenon, here we can only speculate that the signal is related to the change of the free carrier mobility. The change of the mobility can take place at the spin resonance via one of the spin dependent scattering mechanisms. This change of the mobility can lead to the photovoltaic response in a similar way as the change in the carrier density. The effect of the photovoltaic signal amplification due to the modulation of the mobility was already observed in the Shubnikov-de Haas related experiments in III-V HEMTs [6-8]. However, a full quantitative interpretation of our results requires more complete experimental and theoretical developments.

\section{Conclusions}

It may be stated that we observed a new phenomenon in the terahertz photovoltaic response of silicon MOSFETs at low temperature and high magnetic field. The magneto-photovoltaic response exhibits a specific feature moving linearly with the incident radiation frequency. Preliminary studies show possible link between the observed structure in the photovoltaic response and a spin-related phenomenon.

\section{Acknowledgments}

The authors from the University of Montpellier acknowledge support of the region of Languedoc-Roussillon "Terahertz Platform." This work was also supported by GDR-E "Terahertz Sources and Detectors," and by ANR program TeraGaN. We thank M.I. Dyakonov for valuable discussions. The authors from Warsaw acknowledge financial support from Polish Ministry of Higher Education (project No. OR00015509).

\section{References}

[1] R. Tauk, F. Teppe, S. Boubanga, D. Coquillat, W. Knap, Y.M. Meziani, C. Gallon, F. Boeuf, T. Skotnicki, C. Fenouillet-Beranger, D.K. Maude, S. Rumyantsev, M.S. Shur, Appl. Phys. Lett. 89, 253511 (2006).

[2] W. Knap, F. Teppe, Y. Meziani, N. Dyakonova, J. Lusakowski, F. Boeuf, T. Skotnicki, D. Maude, S. Rumyantsev, M.S. Shur, Appl. Phys. Lett. 85, 675 (2004). 
[3] W. Stillman, M.S. Shur, D. Veksler, S. Rumyantsev, F. Guarin, Electron. Lett. 43, 422 (2007).

[4] M. Dyakonov, M. Shur, IEEE Trans. Electron Dev. 43, 380 (1996).

[5] E. Ojefors, U.R. Pfeiffer, A. Lisauskas, H.G. Roskos, IEEE J. Solid-State Circuits 44, 1968 (2009).

[6] M.B. Lifshits, M.I. Dyakonov, Phys. Rev. B 80, 121304(R) (2009).
[7] S. Boubanga-Tombet, M. Sakowicz, D. Coquillat, F. Teppe, N. Dyakonova, W. Knap, K. Karpierz, J. Łusakowski, Appl. Phys. Lett., 95, 072106 (2009).

[8] O.A. Klimenko, Yu.A. Mityagin, H. Videlier, F. Teppe, N.V. Dyakonova, C. Consejo, S. Bollaert, V.N. Murzin, W. Knap,Appl. Phys. Lett 97, 022111 (2010). 